\title{
Continuous chest compressions do not improve outcomes
}

In patients with out-of-hospital cardiac arrest, continuous chest compressions during cardiopulmonary resuscitation (CPR) do not increase rates of survival or favourable neurological function in comparison to interrupted compressions according to findings published in The New England Journal of Medicine and presented at the AHA Scientific Sessions 2015.

Animal models of nonasphyxial arrest and observational studies on individuals with out-of-hospital arrest suggest that the use of continuous compressions results in better neurological function and improved survival rates, respectively, compared with compressions interrupted to provide ventilation. However, no

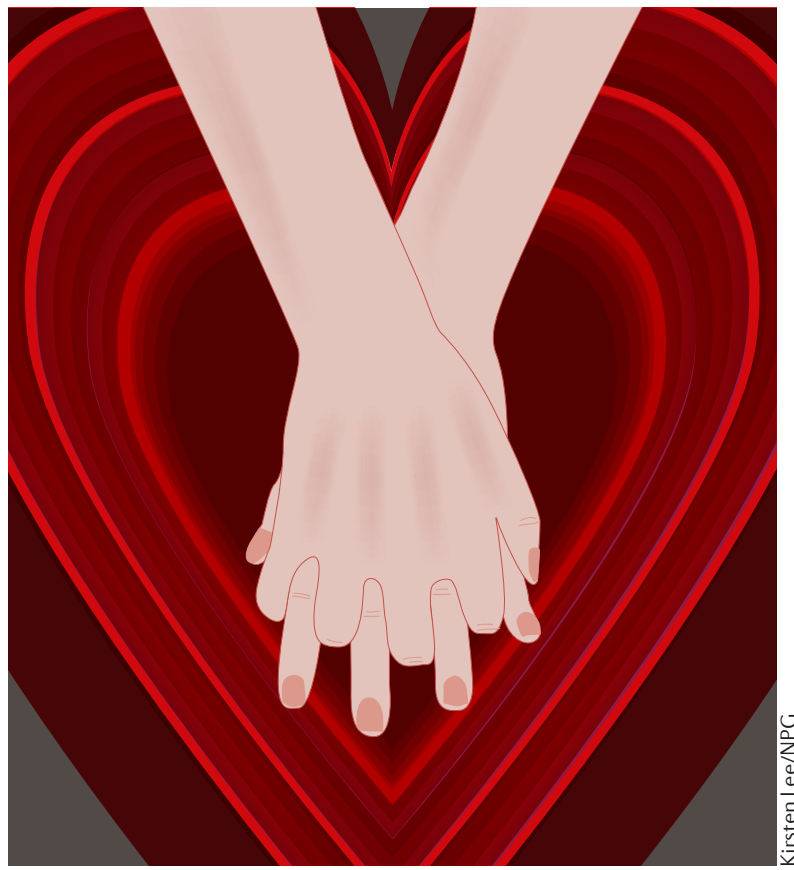

large, randomized trials had been conducted to test these findings.

Accordingly, the Resuscitation Outcomes Consortium conducted a cluster-randomized trial with crossover including 114 emergency medical service (EMS) agencies. Adults who experienced a cardiac arrest out of hospital that was not related to trauma received either continuous compressions (100 per minute) and 10 ventilations per minute given simultaneously (intervention group), or interrupted compressions at a ratio of 30 compressions to two ventilations (control group). Clusters of agencies were randomly assigned to either of these resuscitation strategies and crossed over twice per year to perform the other procedure. The primary outcome was the rate of survival to hospital discharge. Neurological function at discharge (measured using the modified Rankin scale) and hospital-free survival (defined as the number of days alive and out of hospital during the 30 days after arrest) were assessed as secondary outcomes.

A total of 23,711 patients were included. The investigators found that continuous chest compressions did not improve rates of survival in the intervention group (9.0\%) compared with the control group (9.7\%; $95 \% \mathrm{CI}-1.5$ to $0.1, P=0.07)$. Similarly, favourable neurological function was reported in $7.0 \%$ of survivors in the intervention group compared with $7.7 \%$ in the control group (95\% CI -1.4 to 0.1 , $P=0.09)$. Patients receiving continuous chest compressions, however, had significantly fewer days alive and out of hospital during the first month after arrest (95\% CI -0.3 to $-0.1, P=0.004)$. In a per-protocol analysis, significantly fewer of these patients survived to discharge than those who received interrupted compressions (95\% CI -2.9 to $-1.1, P<0.001)$.

"Both the continuous compressions and the interrupted compressions group did well, but it appears that the interrupted compressions group did a bit better. This reinforces the need for EMS providers to continue to ventilate patients," summarizes Graham Nichol, the lead investigator of the trial.

Several factors could have affected these results; for example, the quality of care that patients received between resuscitation and discharge from hospital was measured, but was not mandated. "We are continuing to analyse the data to understand potential mechanisms for the differences between groups," says Nichol. Furthermore, inappropriate levels of oxygenation and hyperventilation have been associated with poor outcomes; however, levels of oxygenation or minutes of ventilation delivered during the event were not measured. "In light of the need for ventilation, we have initiated a large randomized trial to test whether different methods of airway management improve patient care during cardiac arrest," says Nichol.

Clarissa Oeser 